\title{
Host genetic effects upon the early gut microbiota in a bovine model with graduated spectrum of genetic variation
}

\author{
Peixin Fan ${ }^{1,2} \cdot$ Beilei Bian ${ }^{2} \cdot$ Lin Teng $^{1,2} \cdot$ Corwin D. Nelson ${ }^{2} \cdot$ J. Driver $^{2} \cdot$ Mauricio A. Elzo $^{2} \cdot$ Kwangcheol C. Jeong $^{1,2}$
}

Received: 13 June 2019 / Revised: 27 August 2019 / Accepted: 13 September 2019 / Published online: 17 October 2019

(c) The Author(s) 2019. This article is published with open access

\begin{abstract}
Multiple synergistic factors affect the development and composition of mammalian gut microbiota, but effects of host genetics remain unclear. To illuminate the role of host genetics on gut microbiota, we employed animals with a graduated spectrum of genetic variation with minimal environmental influences. We bred 228 calves with linearly varying breed composition from 100\% Angus (Bos taurus) to 100\% Brahman (Bos indicus), as a proxy for genetic variation, and then raised the offspring in the same environment with identical diets. We hypothesized each breed would harbor distinct gut microbiota due to genetic influence. We found that the gut microbiota of preweaning calves at 3 months old is significantly affected by host genetics, profoundly by paternal genome. We also demonstrate that single nucleotide polymorphisms in host mucin-encoding genes, critical for gut mucosal health, are significantly correlated with both breed composition and mucindegrading gut bacteria. We further demonstrate host genetics indirectly changes gut microbiota composition via microbe-microbe interactions. These findings indicate a strong contribution by host genetics in shaping the gut microbiota during early life stages, shedding light on impact of animal breeding on gut microbiota, which is associated with animal growth and health.
\end{abstract}

\section{Introduction}

The diverse commensal bacterial communities in the gastrointestinal (GI) tract of humans and animals provide fundamental functions including regulating immune system development, increasing the host's digestion capabilities, and preventing pathogen colonization [1-3]. Animal gut microbial communities are acquired and shaped dynamically after birth, and sometimes even before birth; they are complex systems that provide health-relevant functions [4-6]. Genetic coevolution between hosts and gut microbes has resulted in specific

Supplementary information The online version of this article (https:// doi.org/10.1038/s41396-019-0529-2) contains supplementary material, which is available to authorized users.

Kwangcheol C. Jeong

kcjeong@ufl.edu

1 Emerging Pathogens Institute, University of Florida, Gainesville, FL 32611, USA

2 Department of Animal Sciences, Institute of Food and Agricultural Sciences, University of Florida, Gainesville, FL 32611, USA nutritional symbiosis such as short-chain fatty acid production and vitamin synthesis $[7,8]$, which indicates the existence of heritable microbiota. In other words, the nutritional benefits to hosts of some gut bacteria are so strong that some microbe taxa appear to be preferentially selected, either through direct contact or via genetic influence mediated by the parental genome. Due to the indispensable roles of heritable bacterial taxa, key modulators such as host genetics may determine host-microbe specificity to maintain microbial communities in the GI tract; bacterial colonization and filtering occur in response to continuous ecological interactions. Exploring how host genotypes modulate gut bacteria composition will deepen our understanding for establishing new targets to lower intestinal disorders and metabolic syndromes by maintaining homeostasis in the GI tract.

Although the degree to which host genetics shape the gut microbiota remains unclear, a growing number of studies across animal species (e.g., human, mouse, chicken, cattle, and swine) have demonstrated the role of host genetics in the gut microbiota composition [9-13]. With recent advanced technology, specifically genome-wide association study (GWAS), multiple associations have been identified between single nucleotide polymorphisms (SNPs) 
genotypes, primarily located in genes associated with host metabolic syndrome and immune disease, and abundance of commensal bacteria [14-16]. However, recently the statistical significance of associations between host SNPs and individual bacterial taxa has been challenged [17], and additional studies report that environmental factors dominate host genetics in shaping the gut microbiota $[18,19]$. The discrepancies in interpreting the role of host genetics and environmental factors might be due to population variation, genetic distance, age, and environmental conditions. The interaction itself between environmental factors and host genetics could also mask host genetic effects in shaping the gut microbiota.

The GI tract is the largest digestive and immune organ. SNPs in the host genes that are associated with metabolic and immune functions are more frequently associated with the prevalence of commensal bacteria in the GI tract [20]. Hence, we hypothesized that animal populations with significantly different genetic backgrounds in metabolism and immune function would harbor readily apparent host genetic effects on gut microbiota compositions. For instance, Angus cattle (Bos taurus), the most common beef breed in the US, have a faster growth rate and better meat quality compared with the Brahman cattle breed (Bos indicus). Brahman cattle are more common in tropical regions due to their immunity traits of being able to resist pathogenic parasites and pathogenic bacterial colonization in their GI tract [21, 22]. They are also known for their superior heat tolerance and ability to utilize low-quality forage [23, 24]. Beef cattle breeding and genetic selection have been conducted using two breeds to generate livestock with desirable phenotypes such as high growth performance and low incidence of disease outbreaks [25-27].

To understand whether host genetics shapes the early gut microbiota and its effects on host phenotypes, we bred our study animals using a unique multibreed Angus-Brahman (MAB) herd; varying from $100 \%$ of one breed, through a gradual, mixed breed composition, to $100 \%$ of another breed. We selected 228 calves from the newborn herd. Selected calves were representative of the gradual change in genetic background, and had an even distribution of age and gender. We raised them in the same environmental conditions and fed them the same diets to minimize nongenetic influences. We then analyzed the calves' gut microbiota composition when they were 3 months old because an animals' early stage of life is critical due to the high incidence of disease outbreaks and rapid growth rate; the significance of the initial gut microbiota in early life stages has also been emphasized due to its role in the education of host immune systems and prolonged effects on the later stages of animal development $[4,28-30]$.

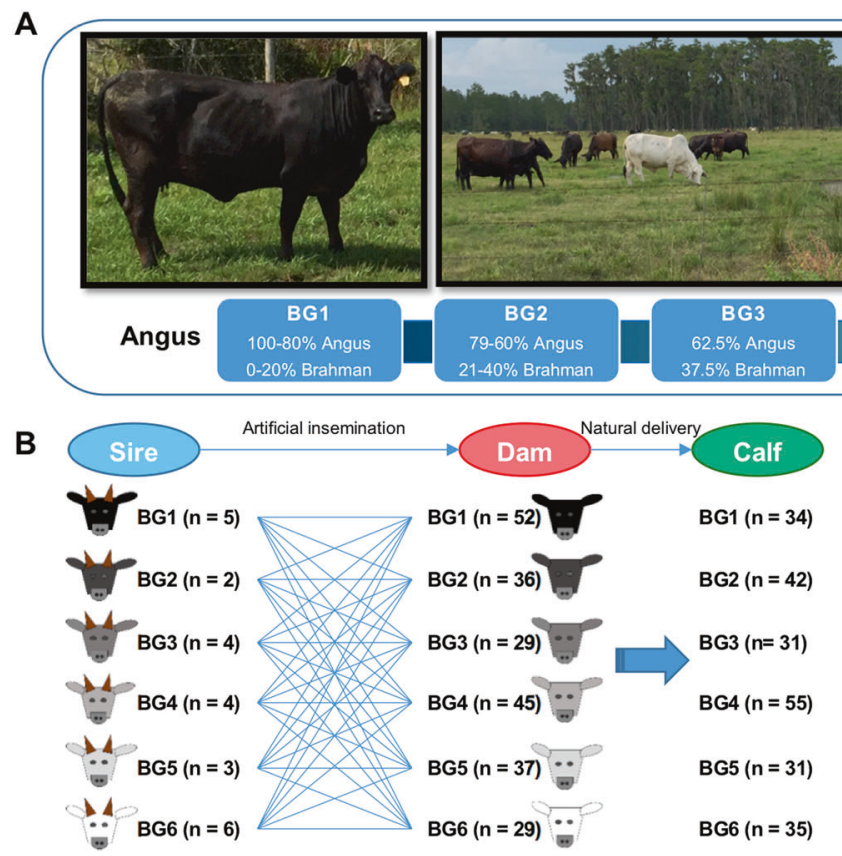

Fig. 1 Animal breeding to generate calves with varying breed composition. a The unique multibreed Angus-Brahman (MAB) herd initiated in 1989 was maintained on the pasture in Florida. Cattle were divided into six breed groups (BGs) based on their breed composition. The breed composition of BG1 to BG6 ranged from 100\% Angus to $100 \%$ Brahman. b Diallel design of mating in the MAB herd where sires from six BGs were mated to dams from six BGs. c Brahman
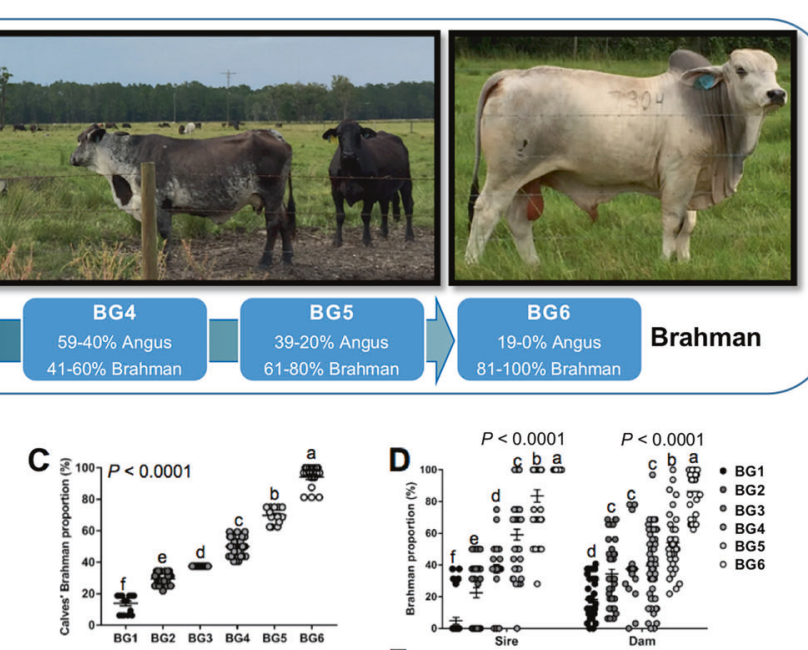

E
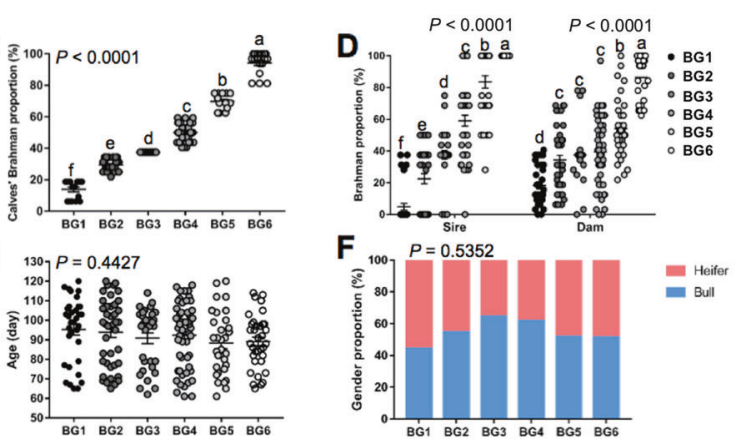

proportion of 228 preweaning calves used in this study. d Brahman proportion of their sires and dams increased from BG1 to BG6. Age (e) and gender distribution (f) were not significantly different among six BGs. In each dotplot or barplot, values that do not have a common superscript are significantly different $(P<0.05)$ based on one-way ANOVA followed by Tukey's HSD test for pairwise comparison of multiple means. c-e The bars represent mean \pm SEM 


\section{Materials and methods}

\section{Ethics statement}

All operations to animals in this study followed the standard practices of animal care and use. The practices related to the animals in this study were approved by the University of Florida Institutional Animal Care and Use Committee (IACUC number 201408629 and 201803744).

\section{Animal genetic background and management}

Preweaning calves in this study were bred from the MAB herd of the University of Florida. The herd was established in 1989 to conduct long-term genetic studies in beef cattle [31]. Calves were assigned to six breed groups (BGs) according to the following breed composition ranges estimated from documented pedigree: $\mathrm{BG} 1=100-80 \%$ Angus and 0-20\% Brahman; BG2 $=79-60 \%$ Angus and 21-40\% Brahman; $\mathrm{BG} 3=62.5 \%$ Angus and $37.5 \%$ Brahman, BG4 $=59-40 \%$ Angus and 41-60\% Brahman, BG5= 39-20\% Angus and 61-80\% Brahman, and BG6 $=19-0 \%$ Angus, and 81-100\% Brahman (Fig. 1a). Mating in the MAB herd followed a diallel design where sires from each of the six BGs were mated to dams from all six BGs [31]. The calves were naturally delivered on the pasture.

The preweaning calves were kept at the Beef Research Unit in Waldo, FL. and were raised with their dams on the same bahiagrass (Paspalum notatum) pastures. Animals in the herd received a complete mineral supplement (UF University Special Hi-Cu Mineral, University of Florida, Gainesville, Florida), and were provided with bermudagrass (Cynodon dactylon) hay and cotton-seed (Gossypium spp.) meal. Calf weights were taken immediately after birth and when fecal samples were collected.

\section{Sample collection and processing}

Fecal and blood samples were collected from 228 preweaning calves $\left(n_{\text {bull }}=126, n_{\text {heifier }}=102\right)$ ranging in age from 60 to 120 days in March and April 2016. Fecal samples were collected as previously described with minor modifications [32]. Briefly, fecal samples were collected from the rectal-anal junction using sterile cotton swabs. Swabs with fecal samples were placed in a $15 \mathrm{~mL}$ conical tube on ice and were transported on the same day to the laboratory for further processing. Each swab sample was resuspended in $2 \mathrm{~mL}$ of Luria-Bertani broth and $2 \mathrm{~mL}$ of $30 \%$ glycerol, split into four $2 \mathrm{~mL}$ tubes and frozen in an ultra-low freezer at $-80^{\circ} \mathrm{C}$. Blood samples $(10 \mathrm{~mL}$ per calf) were collected through the jugular by venipuncture. A portion $(2 \mathrm{~mL})$ of whole blood samples were stored at $-20{ }^{\circ} \mathrm{C}$ for genotyping analysis. Plasma was separated from the remaining blood sample by centrifugation at $1000 \times g$ for $20 \mathrm{~min}$ at $4{ }^{\circ} \mathrm{C}$, and the supernatant was collected and stored at $-20^{\circ} \mathrm{C}$ for biochemical analysis.

\section{S rRNA gene sequencing}

Genomic DNA was extracted from $500 \mu \mathrm{L}$ of each fecal sample using the QIAamp PowerFecal DNA kit according to the manufacturer's instructions (Qiagen, USA). The concentration and purity of the DNA were measured using a Nanodrop instrument (Spectrophotometer ND-1000, Thermo Fisher Scientific, USA). The DNA library was prepared and sequenced as described in the previous study [33]. Briefly, the V4 region of the 16S rRNA gene was amplified by polymerase chain reaction (PCR) with dual-index primers and Pfx AccuPrime master mix (Invitrogen, USA) [33]. The amplicons were purified and normalized in equimolar amounts using the SequalPrep plate normalization kit (Invitrogen, USA). The same amount of barcoded V4 amplicons from each sample were pooled to construct the DNA library. The fragment size and concentration of the DNA library were determined by tape station and Kapa quantitative PCR (qPCR) (Kapa Biosystems, USA). The final DNA library $(600 \mu \mathrm{L} 6 \mathrm{pmol} / \mathrm{L}$ library) was loaded into MiSeq v2, $2 \times 250$ cycle cartridge (Illumina, USA), and was sequenced using the Illumina MiSeq platform.

\section{Microbial community analysis}

Raw sequencing reads were obtained from the Illumina BaseSpace website and analyzed with the Quantitative Insights into Microbial Ecology (QIIME) pipeline (version 1.9.0). Full details on 16S rRNA gene sequencing data analysis are available in Supplementary Information.

\section{Co-occurrence network analysis}

To predict bacteria-bacteria interactions in the gut microbial community, co-occurrence patterns of core bacterial families, and genera that are present in at least $50 \%$ of samples were evaluated in the network interface using pairwise Spearman's rank correlations $\left(r_{\mathrm{s}}\right)$ based on the relative bacterial abundance according to the previous study [34]. The Spearman rank correlation was analyzed using Hmisc within RStudio (version 1.1456). A significant rank correlation between two taxa $\left(r_{\mathrm{s}}>0.2\right.$ or $r_{\mathrm{s}}<-0.2$, FDR-adjusted $P$-value $<0.05)$ was considered as a co-occurrence event. The network was visualized using the Force Atlas algorithm in the interactive platform Gephi (http://gephi.org). In the network, nodes represented different taxa, and edges indicated correlations among nodes. The size of the nodes represented the degree of connection, and the thickness of edges indicated the strength of the correlation. 


\section{Functional prediction of gut microbiome}

Functional capacity of the gut microbial community was predicted using PICRUSt (phylogenetic investigation of communities by reconstruction of unobserved states) online Galaxy version (http://galaxy.morganlangille.com/). The closed reference OTU table was generated by picking OTU against the 13 August 2013 Greengenes database using QIIME (version 1.9.0). Normalization of copy numbers, metagenome prediction, and function categorization based on Kyoto Encyclopedia of Genes and Genomes pathways were conducted using the online Galaxy version on Huttenhower Lab (v1.0.0) servers according to a standard analysis process [35].

\section{Quantitative real-time PCR analysis}

The qPCR was conducted to confirm the differences in the relative abundance of Faecalibacterium prausnitzii and Clostridium perfringens between BG1 and BG6. Full details on the qPCR analysis are available in Supplementary Information.

\section{Animal genotyping}

Animal genotyping was conducted as previously [36]. Briefly, DNA was extracted from calf blood samples using the QIAamp DNA mini kit according to the manufacturer's instructions (Qiagen, USA). DNA samples were genotyped with GeneSeek Genomic Profiler F-250 at Neogen Corporation (GGP F-250, Neogen Genomics, USA). Quality control (QC) was conducted using the software PLINK1.9. QC filters included genotype completion rate $(<90 \%)$, minor allele frequency $(<1 \%)$, genotype call rate $(<90 \%)$, and Hardy-Weinberg equilibrium deviation (chi-square $P$-value $<$ $\left.10^{-8}\right)$. After removing samples with low genotype completion rate $(<90 \%), 220$ out of 225 samples were available for subsequent analysis. From an initial set of 221,049 SNPs, 77,948 autosomal SNPs passed QC filters and were used for principal component analysis (PCA). The PCA was conducted with the input of SNP genotyping matrix (0: reference homozygous, 1: heterozygous variant, 2: homozygous variant, 5: missing) using the prcomp function of RStudio (version 1.1456). The correlation between the Brahman proportion estimated by pedigree and top $\mathrm{PC}(\mathrm{PC} 1)$ was evaluated by Pearson's correlation coefficient.

\section{Detection of blood parameters}

The plasma glucose and nonesterified fatty acids (NEFA) concentrations were determined using glucose kit and NEFA kit (Randox Laboratories Ltd, UK), respectively. An automated RX series Clinical Chemistry Analysers
(Randox Laboratories Ltd, UK) was used for all measurements. The plasma IgG1 concentrations were detected using a bovine IgG1 ELISA Quantitation set (Bethyl Laboratories, USA) according to the manufacturer's protocol. Plasma was diluted in Tris-buffered saline (TBS)Tween to a final dilution factor of $4 \times 10^{4}$. All dilutions were duplicated. Absorbance was read using a BioTek Synergy plate reader (BioTek Instruments, Inc., USA) at a wavelength of $450 \mathrm{~nm}$.

\section{Statistical analysis}

All statistical analyses were conducted using RStudio (version 1.1456). The normal distribution of variables was assessed using the Shapiro-Wilk's test with the shapiro.test function. The nonnormal values were log-transformed before downstream analysis. For the relative abundance of specific bacterial taxa that were not present in all the samples, a small numeric constant (half of the detection limit: 0.00003663 ) was added to all values before logarithm transformation.

Fold differences in the copy number of $F$. prausnitzii and $C$. perfringens between BG1 and BG6 were analyzed by Student $t$-tests by using the $t$.test function. Differences in age, breed composition, gender among BGs, as well as differences in the relative abundance of mucin-degrading bacteria among calves with different SNP genotypes were analyzed by using the one-way analysis of variance (ANOVA) test followed by Tukey's honestly significant difference (HSD) test for pairwise comparison of multiple means. The aov and TukeyHSD functions were used, respectively. A $P$-value $<0.05$ was considered to be statistically significant, and $0.05<P$-value $<0.1$ was considered as tendency towards significance.

A multiple linear regression model was applied to analyze the fixed effects of breed composition, age, and sex on response variable including Chao 1, Shannon index, the relative abundance of core bacteria, microbial function, weight gain, and blood parameters. Associations between SNP genotypes and the relative abundance of bacteria were accessed using a multiple linear regression model with the fixed effects of Brahman proportion, SNP genotype, age and sex, and the dependent variable of relative abundance of mucin-degrading bacteria. The potential contribution of bacteria on animal phenotypes was assessed using a multiple linear regression model with the fixed effects of the relative abundance of bacteria, Brahman proportion, age and sex, and the dependent variable of weight gain and blood parameters. The glm function was used to fit the generalized linear models. The Akaike information criterion was used to choose the best model.

Correlations between average of Brahman proportion in each BG and prevalence of OTU in each BG were 

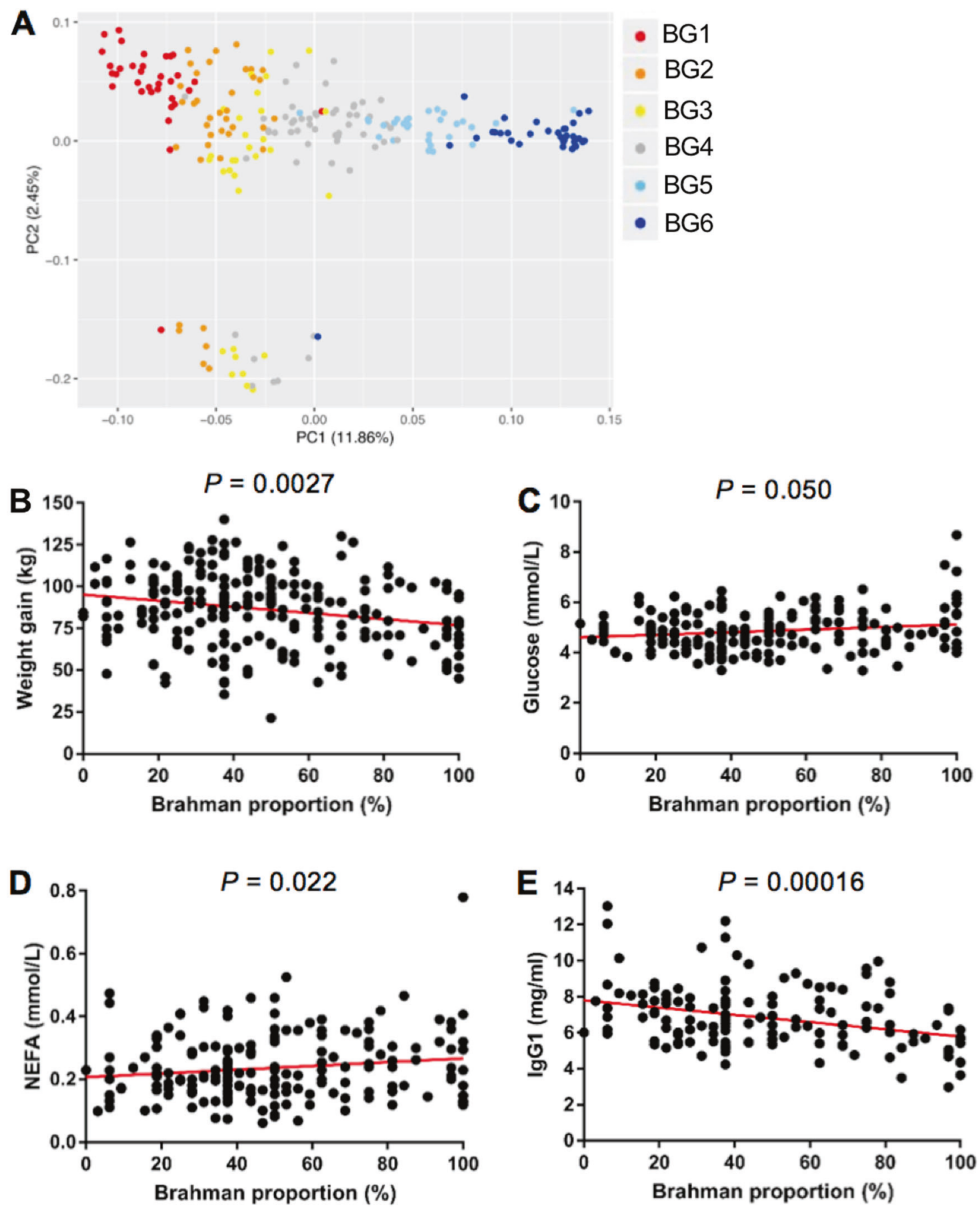

Fig. 2 Genotype and phenotype of the generated calves. a The PCA plot based on SNP genotyping data shows the genetic distance of the calves across six BGs. The color of the dots represents the BG of calves estimated by pedigree. $\mathbf{b}$ Weight gain of the preweaning calves was negatively correlated with calves' Brahman proportion. c Plasma

assessed by Pearson correlation coefficients. Correlations between Brahman proportion and genotype of SNPs in or near mucin-encoding genes were assessed by Spearman rank correlation coefficients. The correlations were analyzed by using the cor.test function. The $P$-values were glucose level tended to be positively correlated with calves' Brahman proportion. d Plasma nonesterified fatty acid (NEFA) level was positively correlated with calves' Brahman proportion. e Plasma IgG1 level was negative correlated with calves' Brahman proportion. b-e The Brahman proportion was estimated by pedigree

adjusted using the false discovery rate (FDR) method for multiple comparison with the p.adjust function. For Pearson correlation, an FDR-adjusted $P$-value $<0.05$ was considered statistically significant. For the Spearman rank correlation, the significant correlation was considered 
with coefficient $>0.2$ or $<-0.2$, as well as an FDRadjusted $P$-value $<0.05$.

\section{Results}

\section{Animal breeding for generation of a herd with varying breed composition}

To understand the influence of host genetics on early development of gut microbiota composition, we bred calves with different breed composition using a unique MAB herd (Fig. 1a). We selected 228 preweaning calves from a total of 278 newborn calves, based on breed composition, age, and gender. Twenty-four sires from six BGs were mated to 228 dams from the same six BGs, resulting in 228 calves that were naturally delivered (Fig. 1b). All calves were raised in the same environmental conditions on pasture with their dams. The calves were also assigned into six BG based on their breed composition, ranging from 100\% Angus to $100 \%$ Brahman (Fig. 1c). The information of preweaning calves including age, sex, and breed composition, as well as breed composition of their sires and dams is presented in Supplementary Table S1. The Brahman proportion of calves (Fig. 1c), as well as that of their sires and dams (Fig. 1d) gradually increased from BG1 to BG6, with a similar distribution of age ranges (Fig. 1e) and gender (Fig. 1f) across the six BGs.

The gradual change of genetic composition of the study herd was evaluated by measuring genetic distance and physiological parameters (Fig. 2). In the PCA plot, the first and second PCs (PC1 and PC2) explained 11.86\% and $2.45 \%$ of the variation in the entire genetic data, respectively (Fig. 2a). The PC1 had a very strong correlation $(R=$ $\left.0.97, P=2.2 \times 10^{-16}\right)$ with the Brahman proportion estimated by pedigree (Supplementary Fig. 1), indicating the strong agreement on population structure between estimation from pedigree and evaluation by SNP genotyping.

For physiological analysis, a multiple linear regression model was applied including Brahman proportion, age in days, and gender as three independent variables, and phenotypes including weight gain, glucose, NEFA, and IgG1 concentrations as dependent variables. All the phenotypes considered in this study were significantly associated with breed composition, or tended to be (Fig. 2b-e). Calves with higher Brahman proportion gained less weight (Fig. 2b, $P=0.0027$ ), and had higher plasma glucose (Fig. 2c, $P=$ 0.050 ) and NEFA levels (Fig. $2 \mathrm{~d}, P=0.022$ ). These data are consistent with previous studies which show slower growth rate and higher energy expenditure of Brahman calves compared with Angus calves [37, 38]. In addition, we observed less plasma IgG1 (Fig. 2e, $P=0.0002$ ) level in calves with higher Brahman proportion, indicating variation in systemic immune function among the calves with different breed composition. This may support the previous observation that the Bos indicus breed is more resistant to parasites compared with the Bos taurus breed partly due to the distinct immune signature in the skin [39]. Taken together, these data indicate that we generated calves belonging to six BGs with varied breed composition that was consistent with the measured phenotypes.

\section{The gut microbiota composition differs with breed composition of calves}

To characterize the early gut microbiota of MAB calves, the $16 \mathrm{~S}$ rRNA gene sequencing was conducted. An average of $114,771 \pm 2917$ (mean \pm SEM) raw paired-end raw reads were generated per fecal sample, clustering into $40850 \pm$ 756 (mean \pm SEM) OTUs, ranged from 13,656 to 81,888 (Supplementary Table S2). The sequencing depth was normalized to 13,650 per sample for downstream analysis.

Although the alpha diversity measured by Shannon index was similar among six BGs (Fig. 3a, $P>0.05$ ), the beta diversity measured by weighted UniFrac distances accounting for dissimilarity in both presence and abundance of bacteria in the GI tract was significantly different among the six BGs (Fig. 3b, $P=0.047$ ). As shown in the PCoA plot, PC1 and PC2 explained a $40 \%$ variation in the gut microbiota composition among 228 calves (Fig. 3b). BG6 that has the farthest genetic distance showed clear separation with BG1 in the PCoA plot (Supplementary Fig. 2E). However, closer genetic BGs, BG2, BG3, BG4, and BG5, showed less separation with BG1 compared with BG6 (Supplementary Fig. 2A-D), suggesting different gut microbiota structure affected by a gradual change in breed composition.

To identify specific bacteria affected by breed composition, correlations between breed composition and the prevalence as well as the relative abundance of bacteria were analyzed. Among the 734 OTUs that were present in more than $50 \%$ of samples in at least one BG, prevalence of $20.8 \%$ (153 OTUs) showed significant correlation with breed composition after correction of multiple comparison (Supplementary Table S3, $P_{\text {adjust }}<0.05$ ). There were 78 OTUs classified at least at the family level having higher prevalence in calves with more Angus breed proportion (Fig. 4a), and the other 56 having higher prevalence in calves with more Brahman breed proportion (Fig. 4b). These breed-associated OTUs primarily were belonging to Ruminococcaceae and Bacteroidaceae. To explore the influence of breed composition on the bacterial abundance in the GI tract, a multiple linear regression model was applied with three independent variables: Brahman proportion, age of calf in days, and gender; and one dependent variable was applied with the relative abundance of core bacteria present in at least $80 \%$ of fecal samples. As a result, the relative abundance of classified core bacterial family or 


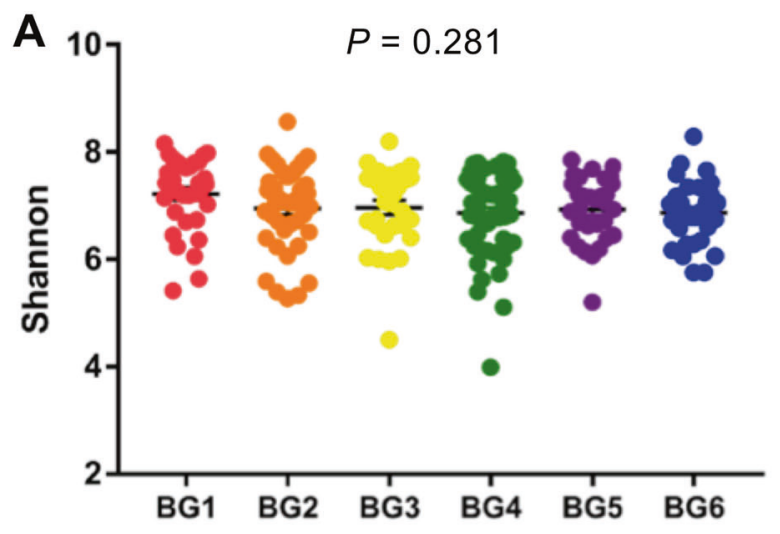

B

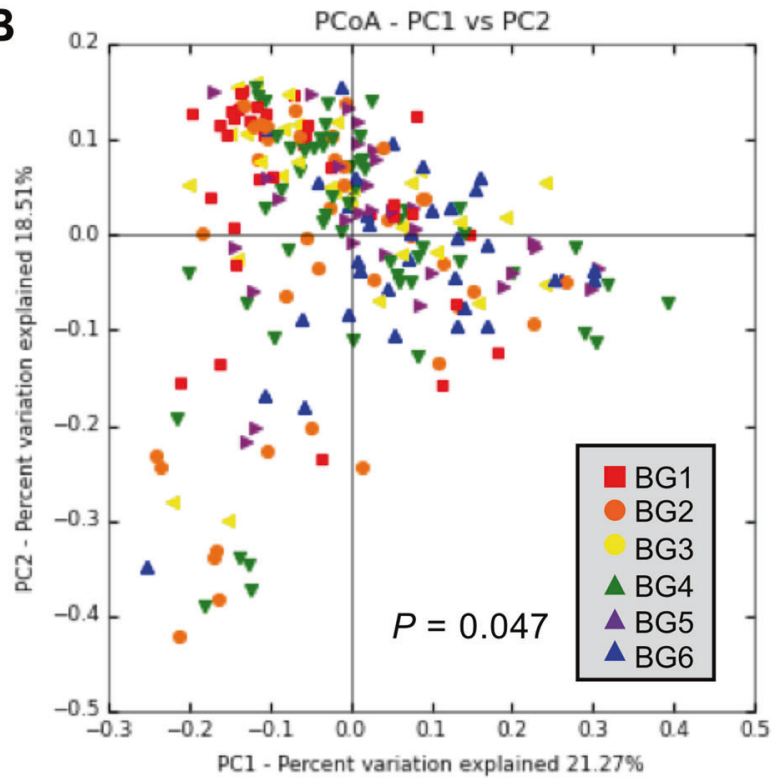

Fig. 3 Alpha and beta diversity of gut microbiota of preweaning calves across six BGs. a Shannon index among six BGs. The difference in Shannon index across six BGs was analyzed using one-way ANOVA followed by Tukey's HSD test for pairwise comparison of multiple means. The bars represent mean \pm SEM. b PCoA plot of weighted UniFrac community distance comparing gut microbiota structure among six BGs. The difference in weighted UniFrac distance among BGs was analyzed by analysis of similarities (ANOSIM)

genus that constituted about $30 \%$ of the microbial community was identified to be significantly linearly associated with breed composition (Fig. 4c and Supplementary Table S4).

Interestingly, bacteria that were enriched in preweaning calves with more Brahman breed proportion included Coprococcus, Faecalibacterium, Blautia, and Butyrivibrio that are fiber-digesting and beneficial butyrate-producing bacteria [40-43]. Consistently, microbial genes involved in carbohydrate metabolism were enriched in preweaning calves with higher Brahman proportion $(P=0.0012)$ predicted by PICRUSt, especially for genes participating in the metabolism of fructose, mannose, galactose, starch, and sucrose (Supplementary Fig. 3). In contrast, bacteria enriched in calves with higher Angus proportion were predicted to have a stronger ability to metabolize lipids and amino acids (Supplementary Fig. 3). Campylobacter and Enterobacteriaceae, which contain species of pathogenic bacteria that commonly trigger calf diarrhea [44, 45], and mucin-degrading bacteria such as Akkermansia, Rikenellaceae, and Clostridium were more abundant in BGs with a higher proportion of Angus breed. Mucin is a crucial component of the gut mucosal barrier [46]. Elevation of mucin-degrading bacteria that use mucin as a source of both carbon and nitrogen has been reported to result in an increased susceptibility to GI pathogens due to a reduction in the intestinal barrier [47].

To validate the differences in the bacterial abundance measured by the 16S rRNA gene sequencing, two bacterial species, $F$. prausnitzii and $C$. perfringens were selected for qPCR confirmation in BG1 and BG6. These two bacteria are representative of butyrate-producing bacteria and opportunistic pathogenic bacteria, respectively. Consistent with the $16 \mathrm{~S}$ rRNA gene sequencing data, the copy number of $F$. prausnitzii in BG6 was eight times higher than BG1 (Fig. 4d, $P=0.006$ ); whereas, the pathogenic bacteria $C$. perfringens in BG6 was about $40 \%$ of BG1 (Fig. $4 \mathrm{e}, P=0.011$ ).

\section{Sire breed composition primarily explains the differences in gut microbiota structure among breed groups of preweaning calves}

The calf herd was maintained on the same pasture throughout the length of this study. Although preweaning calves had access to an identical diet, including supplementary feed, the nutrients in milk provided by dams may have differed due to variations in dam breed composition. Therefore, to explore the effects of sire breed composition, which reflect genetic impact only, we regrouped calves based on their sire breed composition to remove any possible effects of milk nutrient variation caused by dam breed composition. Calves were reassigned into four sire breed groups (S-BGs) based on their sire breed composition (Fig. 5a): S-BG1 (calves bred by sires belonging to BG1), S-BG2\&3 (calves bred by sires belonging to BG2 and BG3), S-BG4\&5 (calves bred by sires belonging to BG4 and BG5), and S-BG6 (calves bred by sires belonging to BG6). To minimize genetic variation caused by dams, calves bred from dams belonging to BG1 or BG6 were excluded from the analysis that resulted in balanced dam breed composition among sire progeny. Therefore, the variation in breed composition among preweaning calves from S-BGs would be primarily due to the differences in sire breed composition (Supplementary Fig. 4A-B), while breed composition of dams among S-BGs were similar (Supplementary Fig. 4C). The alpha diversity showed no significant difference among four S-BGs (Fig. 5b), with similar age range (Supplementary Fig. 4D) and gender (Supplementary Fig. 4E). However, the 


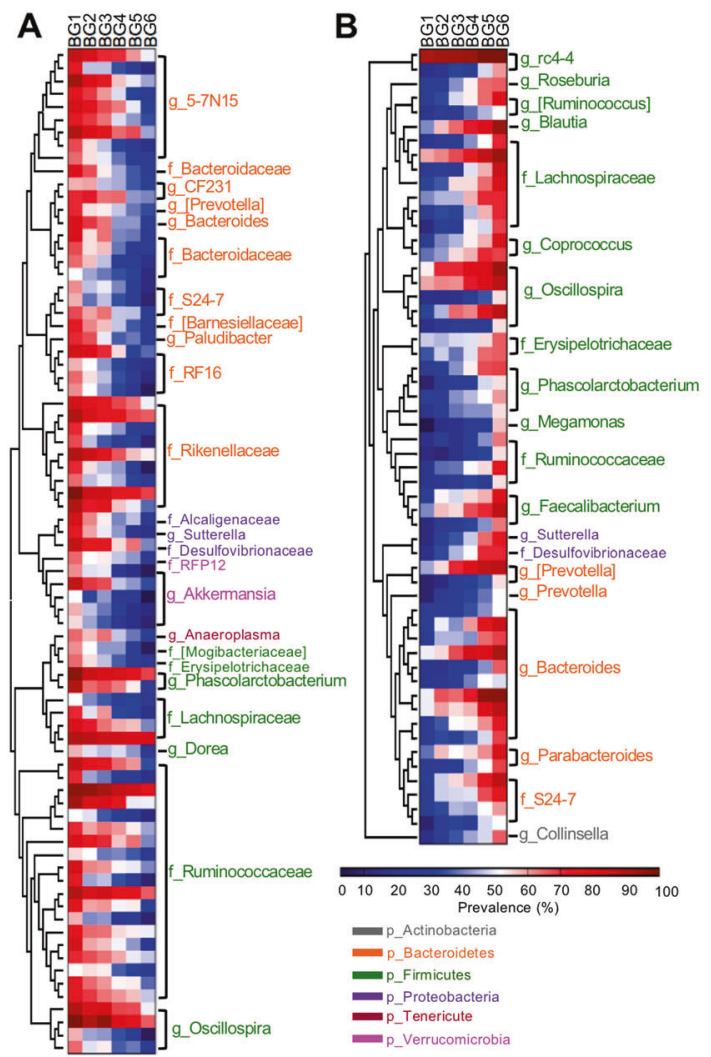

Fig. 4 Gut microbiota composition of preweaning calves across six BGs. a, b Heatmaps represent the prevalence of OTUs across six BGs. Only OTUs that were at least classified at the family level and with their prevalence showing significant positive or negative correlations with Brahman proportion were included in the heatmaps $\left(P_{\text {adjust }}<\right.$ 0.05 ). $\mathbf{c}$ The relative abundances of core bacteria (identified in at least $80 \%$ of the 228 calves) across six BGs. Only bacteria that were linearly

PCoA plot (Fig. 5c) showed that the gut microbiota structure was significantly different among four S-BGs $(P=0.039)$. A multiple linear regression model including the independent variables of calf age, gender, proportion of Brahman sire, and proportion of Brahman dam, and the dependent variable of relative abundances of core bacteria, indicated that $\sim 30-45 \%$ of classified core bacteria were linearly associated with the Brahman proportion of the sire (Fig. 5d, Supplementary Table S5). In addition, the relative abundance of most bacteria that were linearly associated with the Brahman proportion in six calf BGs were also associated with the Brahman proportion in calves of four S-BGs. These bacteria include fiber-digesting bacteria (Coprococcus, Blautia, Faecalibacterium, and Butyrivibrio), and pathogenic bacteria (Clostridium, Campylobacter, and Enterobacteriaceae), as well as mucin-degrading Verrucomicrobia and Rikenellaceae. We also evaluated the effects of sire breed composition on animal phenotypes including weight gain and glucose, NFEA, and IgG1 levels, in four S-BGs but only IgG1 level was tended to show negative association with sire Brahman proportion (Supplementary Table S5, Supplementary Fig. 5A-D). influenced by breed composition, analyzed by a multiple linear regression model, were included in the bar graph. d Fold difference in the copy number of Faecalibacterium prausnitzii between BG1 and BG6. e Fold difference in the copy number of Clostridium perfringens between BG1 and BG6. d, e Data are presented as mean \pm SEM, with statistical differences. $* P<0.05 ; * * P<0.01$

We then explored the effects of dam breed composition on the gut microbiota of the early stage of calves by regrouping calves based on the breed composition of dams (Fig. 5e). To balance sire breed composition of dam progeny, calves bred from sires belonging to BG1 and BG6 were removed, and calves belonging to BG5 and BG6 were combined into one group. Therefore, the variation in breed composition of preweaning calves among five dam breed groups (D-BGs) would be primarily due to the differences in dam breed composition (Supplementary Fig. 6A-C). Surprisingly, unlike dramatic variation in gut microbiota among four S-BGs, the bacterial diversity (Fig. 5f) and microbiota structure (Fig. 5g) among five D-BGs were not significantly different. Only five bacterial families/genera were identified to be significantly linearly associated with dam breed composition based on a multiple linear regression model (Fig. 5h, Supplementary Table S6). However, dam breed composition was significantly associated with animal growth (Supplementary Table S6, $P=0.0002$ ), with calves bred from dams in BG1 having greater weight gain compared with those bred from dams in BG6 (Supplementary Fig. 5E). But no significant 


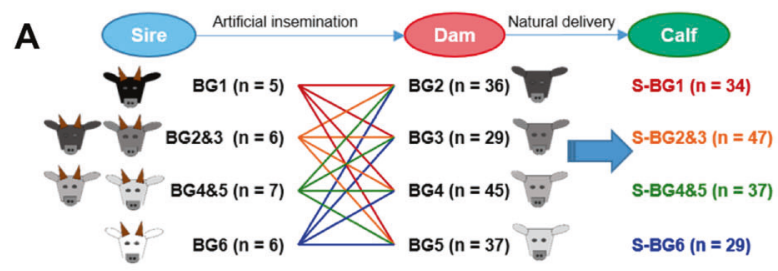

B
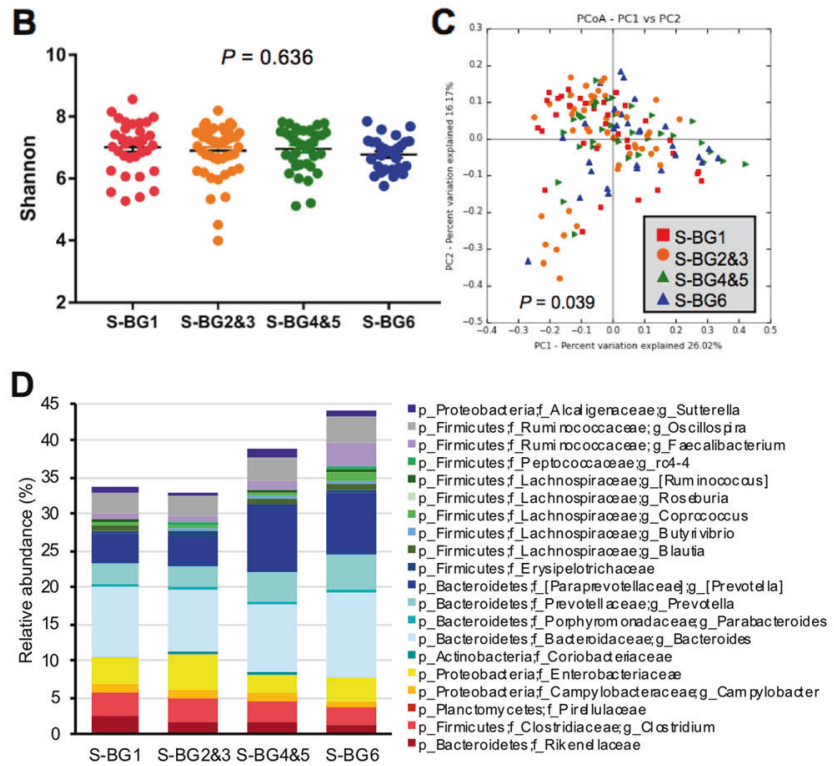

Fig. 5 Sire breed composition mainly explains the linear change of the gut microbiota of preweaning calves among six BGs. a Preweaning calves were regrouped into four sire-BGs based on their sire breed composition. b Shannon index among four sire-BGs. c PCoA plot of weighted UniFrac community distance comparing gut microbiota composition among four sire-BGs (S-BGs). $\mathbf{d}$ The relative abundances of core bacteria that are linearly associated with sire breed

association was observed between dams' breed composition with plasma glucose, NEFA, and IgG1 concentrations (Supplementary Table S6, Supplementary Fig. 5F-H, $P>0.10$ ). Taken together, the breed composition of sires profoundly affected the structure of the early gut microbiota in their progeny, whereas dams' breed composition did not significantly affect the gut microbiota.

\section{SNP genotypes in mucin-encoding genes are associated with breed composition and mucin- degrading bacteria}

As several mucin-degrading bacteria were linearly associated with breed composition, we hypothesized that variations in the genotype of mucin-encoding genes among the calves might contribute to the differences in the relative abundance of mucin-degrading bacteria. Among the 327 SNP markers located in or near mucin-encoding genes identified by genotyping, 173 had known rs ID, call rate higher than $90 \%$ and minor allele frequency higher than $1 \%$, and were used for downstream analysis. Spearman rank correlation coefficients

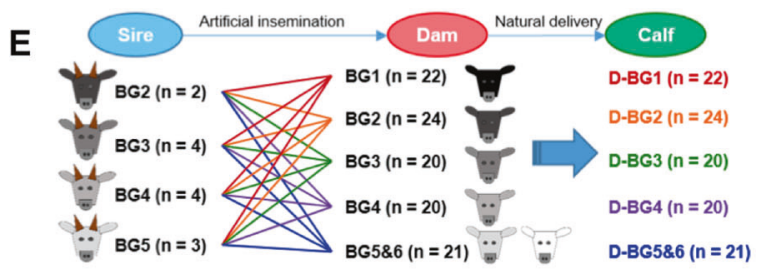

$\mathbf{F}$
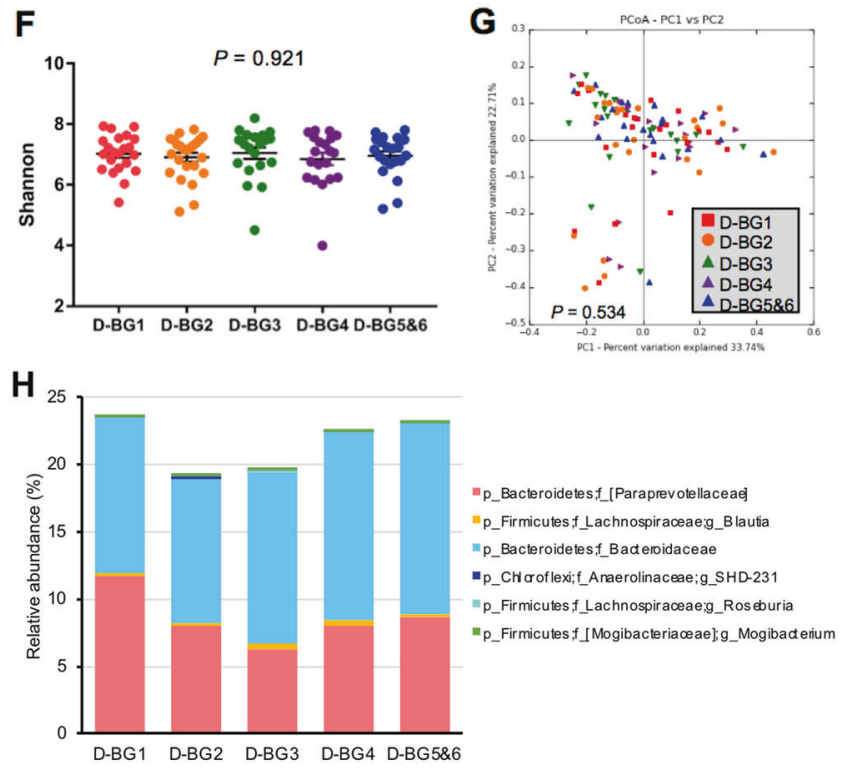

composition. e Preweaning calves were regrouped into five dam-BGs (D-BGs) based on their dam breed composition. f Shannon index among five D-BGs. g PCoA plot of weighted UniFrac community distance comparing gut microbiota composition among five D-BGs. $\mathbf{h}$ The relative abundances of core bacteria that are linearly associated with dam breed composition. b, f The bars represent mean \pm SEM

indicated that genotypes of 108 SNP markers located in or near the mucin-encoding genes were significantly correlated with breed composition (Supplementary Table S7, Fig. 6a, $r_{\mathrm{s}}$ $>0.20$ or $r_{\mathrm{s}}<-0.20, P_{\text {adjust }}<0.005$ ).

To further explore the connection between SNP genotypes in or near mucin-encoding genes and mucin-degrading bacteria, we used a multiple linear regression model including the genotype of 152 breed-associated SNPs, Brahman proportion, age in days and gender as four independent variables, and the relative abundance of three mucin-degrading bacteria that are enriched in calves with more Angus proportion (Clostridium, Rikenellaceae, and Akkermansia) as a dependent variable. We identified 34 significant linear associations between SNP genotype in mucin-encoding genes and the relative abundance of mucin-degrading bacteria (Supplementary Table S8). In Fig. 6b-e, we show difference in relative abundance of mucin-degrading bacteria among the genotypes of four SNPs that had strongest association with bacteria. The SNPs were located in MUC12 (rs43764633), MUC13 (rs134555951), MUC20 (rs208323556), and MUC4 (rs208812969) that are expressed in the bovine GI tract [48]. 
Fig. 6 Genotypes of SNP located in or near mucinencoding genes are associated with breed composition and abundance of mucin-degrading bacteria. a The heatmap represents the linear change of SNP genotypes located the in mucin-coding genes with breed composition. b The $\log _{10}$ transformed relative abundance of Clostridium in preweaning calves who have different genotypes at rs43764633 (MUC12). c-e The $\log _{10}$ transformed relative abundance of Rikenellaceae in preweaning calves who have different genotypes at rs134555951 (MUC13), rs208323556

(MUC20), and rs208812969

(MUC4). In each dotplot, values that do not have a common superscript are significantly different $(P<0.05)$ based on one-way ANOVA followed by Tukey's HSD test for pairwise comparison of multiple means

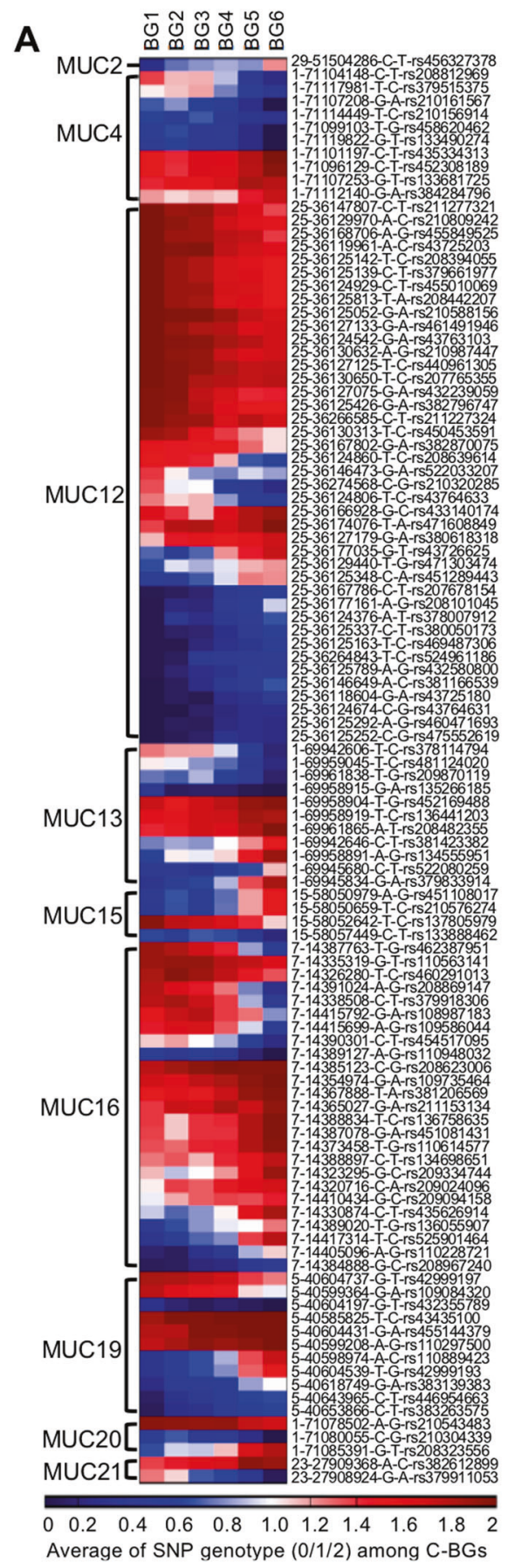

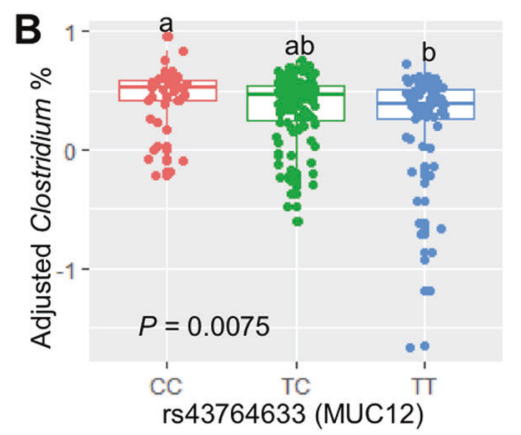
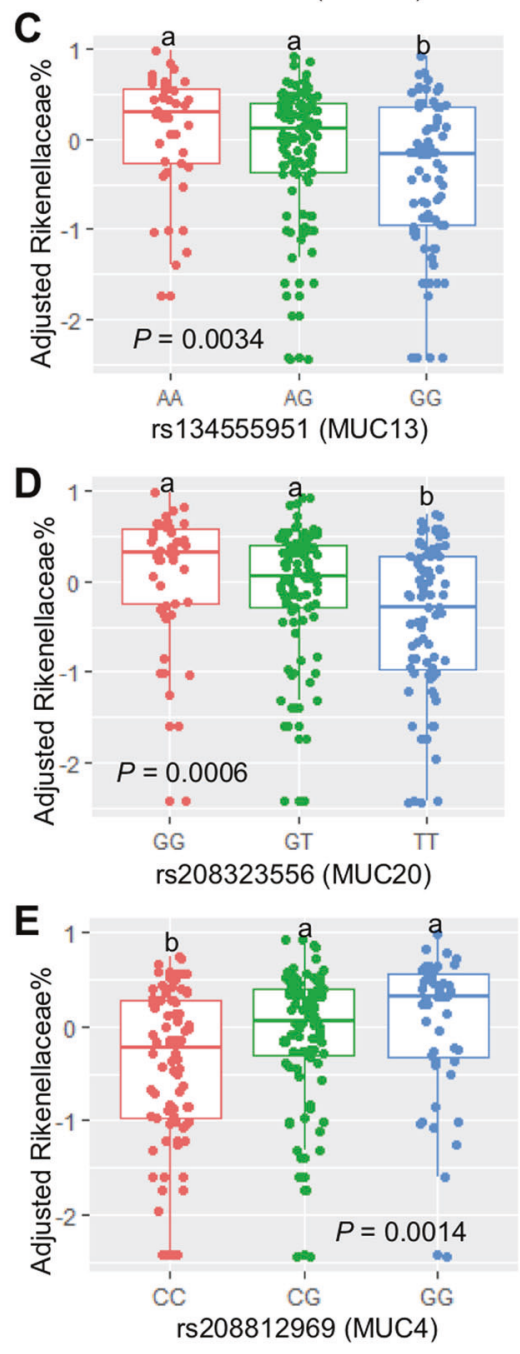

\section{Influence of host genetics on microbe-microbe interactions}

To expand our analysis beyond the selected host-microbe interaction, we analyzed bacteria-bacteria interactions using a co-occurrence network analysis. This analysis was conducted on 57 classified core bacterial families or genera that were present in over $50 \%$ of the fecal samples. A total of 550 connections had significant Spearman rank correlations $\left(P_{\text {adjust }}<0.05, \quad r_{\mathrm{s}}>0.2\right.$ or $\left.r_{\mathrm{s}}<-0.2\right)$ among microbes
(Fig. 7). The strongest positive correlation was detected between Bacillus and Lysinibacillus $\left(r_{\mathrm{s}}=0.8043\right)$, while the strongest negative correlation was between 5-7N15 and Bacteroides ( $r_{\mathrm{s}}=-0.802$ ). Especially, 21, 31, and 32 correlations were detected between mucin-degrading bacteria Clostridium, Akkermansia, and Rikenellaceae with other bacteria, respectively, including negative correlations with butyrate-producing bacteria Blautia, Faecalibacterium, and Coprococcus, as well as positive correlations with opportunistic pathogen Campylobacter. 


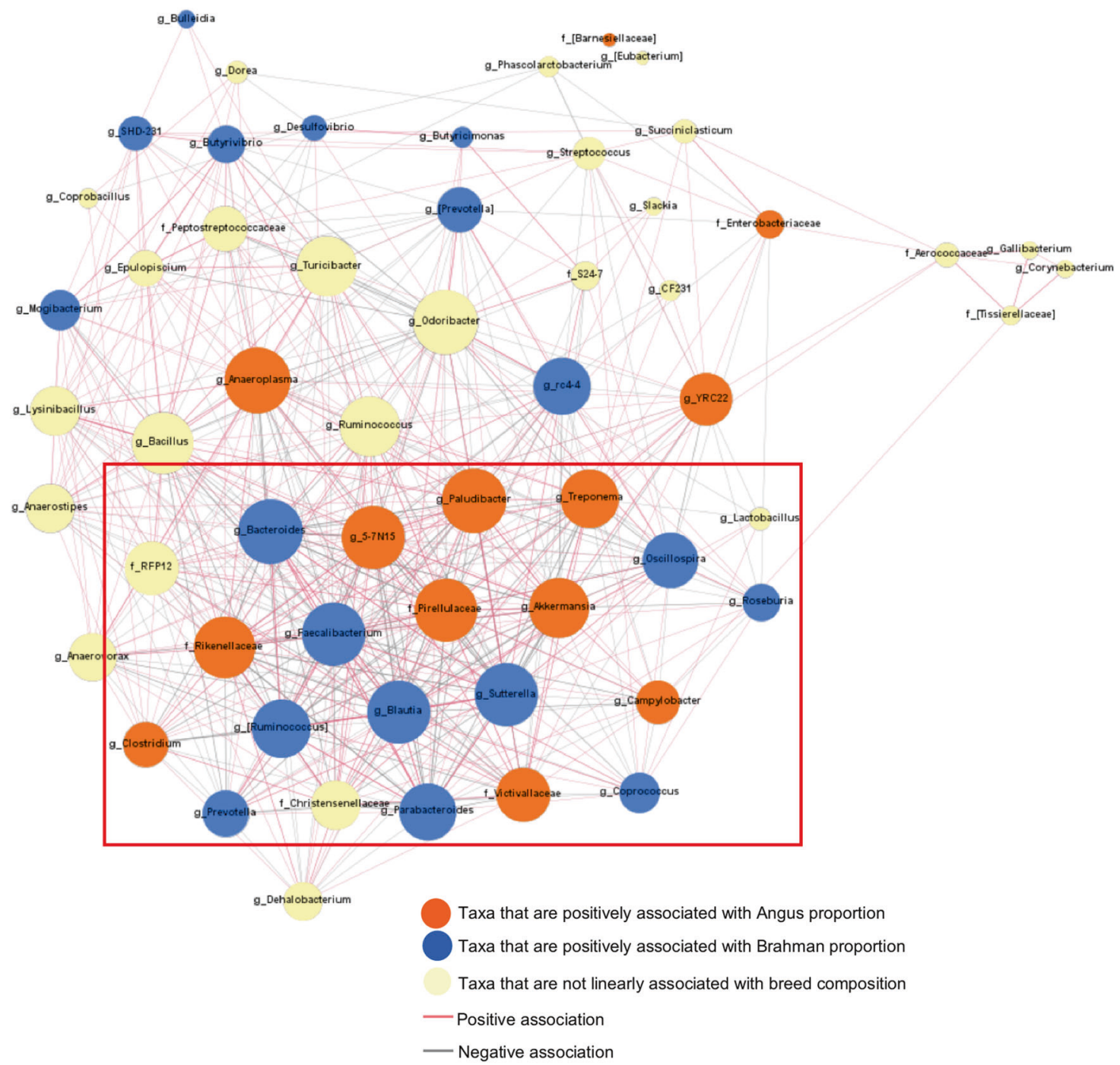

Fig. 7 Co-occurrence bacterial network shows 550 significant connections $\left(P_{\text {adjust }}<0.05, r_{\mathrm{s}}>0.2\right.$, or $\left.r_{\mathrm{s}}<-0.2\right)$ among 57 bacteria families or genera (identified in at least $50 \%$ of 228 calves) in preweaning calves. Connections were detected based on Spearman's rank

\section{Potential contributions of gut microbiota on calves}

To further explore the potential impact of gut microbiota on the MAB herd, we analyzed the associations between the relative abundance of core bacteria and animal growth, metabolic parameters, or immune parameters using the multiple linear regression model. The relative abundance of bacteria served as an explanatory variable along with age, Brahman proportion, and gender. Numerous bacteria, including butyrate-producing bacteria Faecalibacterium, Oscillospira, Blautia that were more abundant in calves with more Brahman proportion were positively associated with weight gain (Fig. 8). Negative associations were detected between weight gain and the relative abundance of Bacteroidaceae, Peptostreptococcaceae, Clostridiaceae, Bacillus, and Streptococcus, likely containing opportunistic correlation coefficient. Dot size represents the number of connections with other taxa. Thickness of lines represent the strength of the relatedness. Genera associated with breed composition are presented in green box

pathogenic bacteria (Fig. 8). In addition, we identified several positive associations between Firmicutes bacteria and plasma glucose level, and negative associations between Bacteroidetes bacteria and plasma NEFA level. However, fewer and weaker associations were detected between the relative abundance of bacteria and plasma IgG1 level (Fig. 8).

\section{Discussion}

To understand the impact of host genetics on the gut microbiota structure, we bred a very unique animal model with a linear change of breed composition and raised them in the same environmental conditions with the same diets to minimize nongenetic influences. By using this animal model, we 


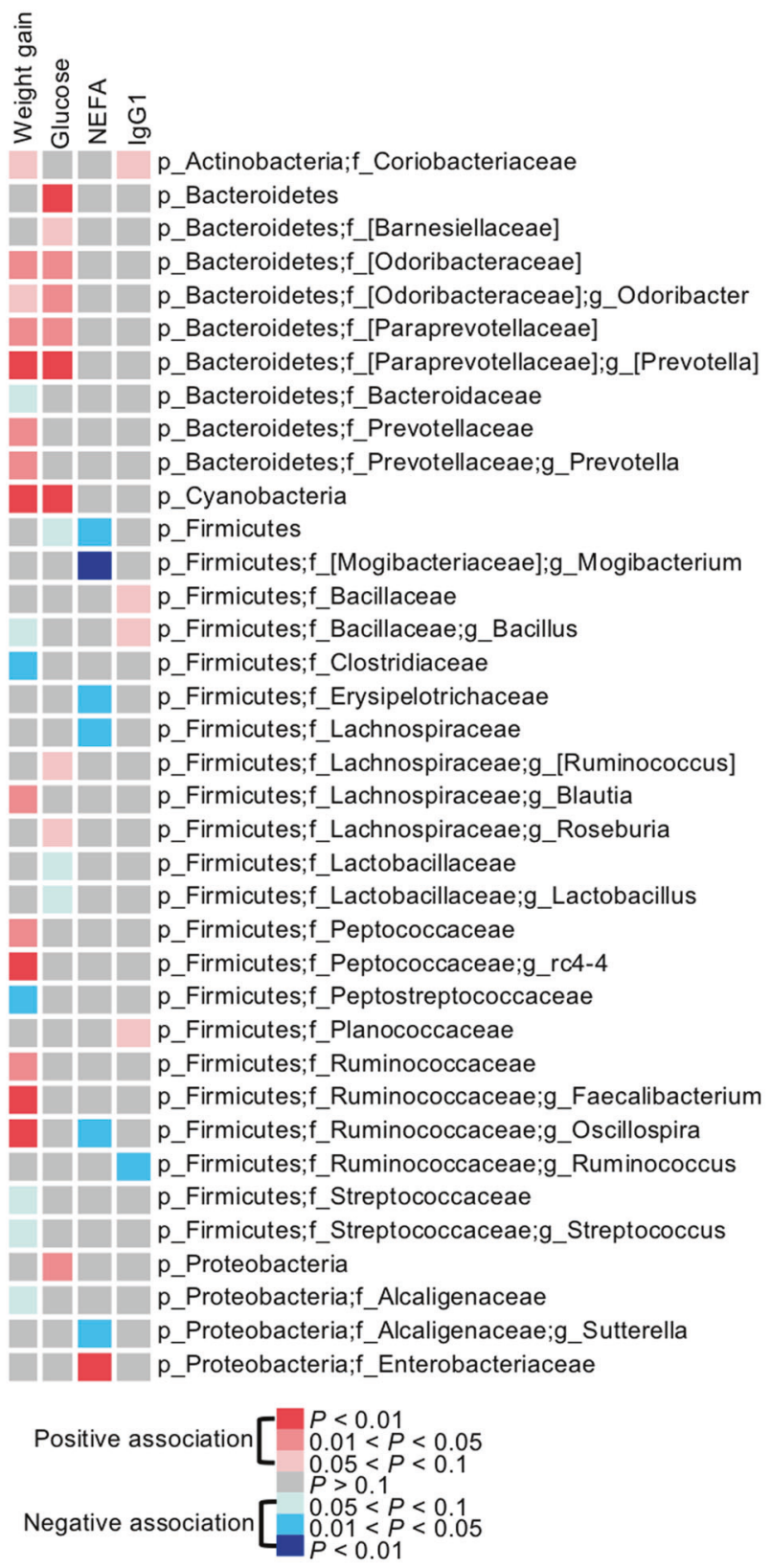

Fig. 8 A heatmap shows association between the relative abundance of core bacteria and weight gain, plasma glucose, NEFA, and IgG1 concentrations analyzed by a multiple linear regression model

observed effects of host genetics on shaping the gut microbiota structure.

The influence of host genetics on gut microbiota was proposed in the early 1980s by observing more similar fecal microbiota shared between five pairs of monozygotic twins than between dizygotic twins using a culture method [49]. Later, with the development of next-generation sequencing and larger sample sizes of twin studies, the role of host genetics in the gut microbiota structure was confirmed, followed by detection of associations between host SNPs and bacterial abundance $[9,14,16]$. However, the level of strength of host genetic impact on the gut microbiota has not reached consensus yet, primarily due to the different populations used in the studies, with variations in genetic distance, as well as other factors such as age, diet, and living style [17, 18, 50-52]. These factors might have masked the significance of the host genetic role in the gut microbiota in previous studies, leading to a failure in the identification of repeatable associations between host SNPs and bacterial abundance.

In this study, we found core microbial genera among MAB calves that were linearly influenced by breed composition and consistent with measured phenotypes. Calves with more Brahman proportion harbored less mucindegrading bacteria and opportunistic pathogenic bacteria, but more butyrate-producing bacteria, which are commonly considered as beneficial commensal bacteria primarily due to their antiinflammatory property. Surprisingly, when we regrouped calves based on sires' breed composition to minimize the maternal effects (e.g., milk composition, companionship), we found that sire genetics had significant impacts in the early gut microbiota structure. However, this same effect was not observed when the calves were regrouped based on their D-BGs. Therefore, consistent associations between the relative abundances of many bacteria in calf BGs and S-BGs indicate that host genetics, influenced heavily by their sires, affect the formation of early gut microbiota structure at the age of 3 months. Previous studies have revealed the effect of sire breed on the rumen microbial populations of beef cattle in feedlot, which is associated with rumen fermentation and methane emissions $[12,53]$. This study reports the strong sire breed effect on hindgut microbiota and growth performance of beef cattle during early stage of life. Further research is needed to determine how long this strong effect continues into later developmental stages, as well as how low abundant bacterial taxa, neglected in this study but could be biologically important, may be influenced by host genetics.

The lesser effect of the dam breed composition on the calves' GI tract microbiota development was quite surprising. Although the relative abundance of several bacteria showed a linear association with dam breed composition, the number was significantly lower compared with that affected by sire breed composition. However, it is noteworthy that dam breed effects may be much more complex. Although we regrouped calves to minimize the maternal effects driven by host genetics, other maternal effects such as milk composition and companionship might have masked potentially undetected genetic effects. Furthermore, genetic relationships among calves in D-BGs could be more complicated compared with that of calves in S-BGs because more dams were used for the mating that might have resulted in higher genetic variability among calves within D-BGs. 
One of the primary functions of the gut microbiota is protecting the gut lining from pathogen colonization. Interestingly, among the bacteria that were linearly associated with breed composition, pathogenic bacteria $C$. perfringens and Campylobacter were less abundant in calves with more Brahman proportion. C. perfringens and Campylobacter are both identified as causes of enteric diseases in young calves due to toxin production [54-56]. Moreover, we also observed that the relative abundance of several mucin-degrading bacteria reduced as Brahman proportion decreased. An increase in mucin-degrading bacteria was reported to be positively associated with pathogen colonization due to the consumption of mucin, which is a crucial component of the intestinal epithelial barrier [47, 57-59]. As expected, positive associations between mucindegrading bacteria and pathogenic bacteria were detected in our study. We further observed a linear association between SNP genotypes located in or near mucin-encoding genes and breed composition, as well as strong correlations between mucin-degrading bacteria (Clostridium, Rikenellaceae, and Akkermansia) and SNPs markers located in mucin-coding genes (MUC4, MUC12, MUC13, and MUC20), which shed light on variation of mucin-degrading bacteria in the MAB herd. Transcription of the four mucin genes has been detected in the GI tract of cattle, including the large intestine [48]. In human studies, membrane-bound MUC4 protects colonic epithelium [60]. MUC12 and MUC20 are involved in the epithelial cell protection [61], and the significant downregulation of MUC12 and MUC20 in the colon and ileum has been detected in patients with Crohn's disease [62]. MUC13 has been reported to be highly expressed on the human intestinal mucosal surface, and polymorphism in MUC13 is related to inflammatory bowel disease [63]. Therefore, the polymorphisms in cattle mucin genes probably contribute to variation in host defense systems among the calves with different breed compositions and result in the distinction of the gut microbiota according to their genetic background.

In this study, we primarily focused on understanding the impact of the gut microbiota in the early preweaning stage in which the rumen is not fully developed yet [64], and consequently, the hindgut is critically vital for energy harvest [65]. In addition, at this stage, animals grow faster and immunity starts to develop, and the GI tract microbiota is more diverse than late growth stage [66]. Based on this evidence, we hypothesized that host genetics probably exert a stronger impact on early gut microbiota that further contributes to animal development. Compared with Angus cattle, Brahman cattle have a greater ability to utilize lowquality feeds that contain low concentrations of protein and soluble carbohydrates [23]. Conversely, Angus cattle grow more quickly, and their meat contains more marbling than Brahman [67]. We found that fiber-digesting bacteria
F. prausnitzii, Blautia producta, Oscillospira, and Coprococcus, considered as beneficial commensal gut bacteria in calf and humans due to their production of butyrate [68-71], increased linearly as Brahman proportion increased. Notably, butyrate-producing bacteria more abundant in calves with more Brahman proportion showed positive associations with weight gain, indicating their contribution on energy harvest during the early stage of calves. Our results also revealed variations in metabolic status among MAB calves reflected in weight gain, plasma glucose, and NEFA concentrations. As predicted by PICRUSt, which has high agreement with metagenomic sequencing data [35], the relative abundances of microbial genes involved in carbohydrate metabolic pathways linearly increased as Brahman proportion increased, while those participating in amino acid and lipid metabolic pathways increased as Angus proportion increased, suggesting that breed composition influenced nutrient environment provided to the gut commensal bacteria.

We also found that the level of plasma $\operatorname{IgG1}$, which is the primary antibody in the circulation system, decreased linearly as calves' and sires' Brahman proportion increased, indicating a linked effect of host genetics on systemic immunity. IgG1 is induced when the infectious disease is manifest to protect the host by binding itself to pathogens [72]. Therefore, calves with greater Brahman proportion potentially had lower IgG1 because they harbored less pathogenic bacteria in the GI tract. Although Bos indicus is known as a disease resistant breed and widely used in animal breeding in the tropical area, most studies focus on parasite resistance due to their thick skin with few explorations in the distinction of their immune function and its relationship with other infectious diseases [22, 72, 73]. The discovery of variation in genotype of the mucin genes, expression of plasma $\operatorname{IgG1}$, as well as the abundance of pathogenic bacteria in calf BGs suggests that the Bos indicus breed may have different innate and adaptive immunity compared with the Bos taurus breed that enables them to better resist infectious diseases.

Besides breed composition, we also assessed the effect of age and gender on gut microbiota structure of preweaning calves by using a multiple linear regression model. Consistent with the previous findings that Brahman cattle reach puberty later than Angus cattle [38, 74], we observed that most bacteria that exhibited linear correlations with Brahman proportion had opposite correlations with calf age (Supplementary Table S4), indicating that the gut microbiota of calves with higher Brahman proportion also develops more slowly than that of calves with higher Angus proportion. Sex of the calf did not significantly influence the gut microbiota structure in this study (Supplementary Fig. $7, P=0.830$ ), but was associated with the relative abundance of several bacteria (Supplementary Table S4). The relative abundance of Bacteroidetes and [Paraprevotellaceae], which was associated 
with plasma glucose level, was higher in heifers, suggesting that gender could partly affect calf growth and metabolic status through changes in microbiota. This is consistent with the previous findings that bacteria associated with sex were involved in numerous carbohydrate and lipid metabolic pathways $[75,76]$.

Furthermore, we found that breed-associated bacteria, such as Anaeroplasma, Paludibacter, Bacteroides, 5-7N15, Pirellulaceae, interacted with many other bacteria, suggesting that the relative abundance of these bacteria were highly dependent on other bacteria in the GI tract. Besides, various negative connections were detected among Angusassociated bacteria and Brahman-associated bacteria, especially among butyrate-producing bacteria (Faecalibacterium, Blautia, Oscillospira) and mucin-degrading bacteria (Clostridium, Rikenellaceae, and Akkermansia). These data are consistent with the previous study reporting the competition between fiber-digesting bacteria and mucindegrading bacteria [47]. Therefore, the host genotype may directly affect on colonization of certain bacteria and indirectly shape the gut microbiota structure through the bacteria-bacteria interactions.

In summary, we found that host genetics, especially from the sire, significantly contribute to the structure of a calf's gut microbiota at age 3 months old. Furthermore, we identified host SNPs that were associated with specific bacterial genera involved in the gut health and nutritional acquisition. Further studies to understand additional factors beyond host genetics, age, and gender are needed to distinguish the factors that affect development of the bovine microbiota structure. Ultimately, understanding mechanisms to develop and maintain the gut microbiota homeostasis will be necessary for a sustainable agricultural production system. These results also have implications for studying the complex suite of factors that lead to human metabolic syndromes and intestinal disorders.

\section{Data availability}

The V4 region of $16 \mathrm{~S}$ rRNA gene sequencing data generated and analyzed during the current study are available in the NCBI primary data archive (PDA) with the accession number SRP115548.

Acknowledgements The authors would like to thank Zhengxin Ma, Shinyoung Lee, Emily Pulliam, and Mercedes Rivera for their help with collection and processing of the fecal and blood samples. This material is based upon work that is supported by the National Institute of Food and Agriculture, U.S. Department of Agriculture, under the award number 2015-68003-22971 to KCJ.

Author contributions PF, MAE, CN, and KCJ designed the study. PF, LT, JDD collected the samples. PF and BB performed the analyses. $\mathrm{PF}, \mathrm{CN}$, MAE, and KCJ wrote the paper. KCJ acquired funding.

\section{Compliance with ethical standards}

Conflict of interest The authors declare that they have no conflict of interest.

Publisher's note Springer Nature remains neutral with regard to jurisdictional claims in published maps and institutional affiliations.

Open Access This article is licensed under a Creative Commons Attribution 4.0 International License, which permits use, sharing, adaptation, distribution and reproduction in any medium or format, as long as you give appropriate credit to the original author(s) and the source, provide a link to the Creative Commons license, and indicate if changes were made. The images or other third party material in this article are included in the article's Creative Commons license, unless indicated otherwise in a credit line to the material. If material is not included in the article's Creative Commons license and your intended use is not permitted by statutory regulation or exceeds the permitted use, you will need to obtain permission directly from the copyright holder. To view a copy of this license, visit http://creativecommons. org/licenses/by/4.0/.

\section{References}

1. Round JL, Mazmanian SK. The gut microbiota shapes intestinal immune responses during health and disease. Nat Rev Immunol. 2009;9:313-23.

2. Maslowski KM, Mackay CR. Diet, gut microbiota and immune responses. Nat Immunol. 2011;12:5-9.

3. Kamada N, Chen GY, Inohara N, Nunez G. Control of pathogens and pathobionts by the gut microbiota. Nat Immunol. 2013;14:685-90.

4. Matamoros S, Gras-Leguen C, Le Vacon F, Potel G, de La, Cochetiere MF. Development of intestinal microbiota in infants and its impact on health. Trends Microbiol. 2013;21:167-73.

5. Lee WJ, Hase K. Gut microbiota-generated metabolites in animal health and disease. Nat Chem Biol. 2014;10:416-24.

6. Neu J. The microbiome during pregnancy and early postnatal life. Semin Fetal Neonatal Med. 2016;21:373-9.

7. Morrison DJ, Preston T. Formation of short chain fatty acids by the gut microbiota and their impact on human metabolism. Gut Microbes. 2016;7:189-200.

8. LeBlanc JG, Milani C, de Giori GS, Sesma F, van Sinderen D, Ventura M. Bacteria as vitamin suppliers to their host: a gut microbiota perspective. Curr Opin Biotech 2013;24:160-8.

9. Goodrich JK, Waters JL, Poole AC, Sutter JL, Koren O, Blekhman R, et al. Human genetics shape the gut microbiome. Cell. 2014;159:789-99.

10. Zhang CH, Zhang MH, Wang SY, Han RJ, Cao YF, Hua WY, et al. Interactions between gut microbiota, host genetics and diet relevant to development of metabolic syndromes in mice. Isme J. 2010;4:312-3.

11. Zhao L, Wang G, Siegel P, He C, Wang H, Zhao W, et al. Quantitative genetic background of the host influences gut microbiomes in chickens. Sci Rep. 2013;3:1163.

12. Roehe R, Dewhurst RJ, Duthie CA, Rooke JA, McKain N, Ross $\mathrm{DW}$, et al. Bovine host genetic variation influences rumen microbial methane production with best selection criterion for low methane emitting and efficiently feed converting hosts based on metagenomic gene abundance. PLoS Genet. 2016;12:e1005846.

13. Camarinha-Silva A, Maushammer M, Wellmann R, Vital M, Preuss S, Bennewitz J. Host genome influence on gut microbial composition and microbial prediction of complex traits in pigs. Genetics. 2017;206:1637-44. 
14. Bonder MJ, Kurilshikov A, Tigchelaar EF, Mujagic Z, Imhann F, Vila AV, et al. The effect of host genetics on the gut microbiome. Nat Genet. 2016;48:1407-12.

15. Wang J, Thingholm LB, Skieceviciene J, Rausch P, Kummen M, Hov JR, et al. Genome-wide association analysis identifies variation in vitamin $\mathrm{D}$ receptor and other host factors influencing the gut microbiota. Nat Genet. 2016;48:1396-406.

16. Goodrich JK, Davenport ER, Beaumont M, Jackson MA, Knight $\mathrm{R}$, Ober $\mathrm{C}$, et al. Genetic determinants of the gut microbiome in UK twins. Cell Host Microbe. 2016;19:731-43.

17. Wen CL, Yan W, Sun CJ, Ji CL, Zhou QQ, Zhang DX, et al. The gut microbiota is largely independent of host genetics in regulating fat deposition in chickens. Isme J. 2019;13:1422-36.

18. Rothschild D, Weissbrod O, Barkan E, Kurilshikov A, Korem T, Zeevi D, et al. Environment dominates over host genetics in shaping human gut microbiota. Nature. 2018;555:210-5.

19. Carmody RN, Gerber GK, Luevano JM Jr., Gatti DM, Somes L, Svenson KL, et al. Diet dominates host genotype in shaping the murine gut microbiota. Cell Host Microbe 2015;17:72-84.

20. Chassaing B, Kumar M, Baker MT, Singh V, Vijay-Kumar M. Mammalian gut immunity. Biomed J. 2014;37:246-58.

21. Jeon SJ, Elzo M, DiLorenzo N, Lamb GC, Jeong KC. evaluation of animal genetic and physiological factors that affect the prevalence of Escherichia coli $\mathrm{O} 157$ in cattle. PLoS One. 2013;8: e55728.

22. Glass EJ, Preston PM, Springbett A, Craigmile S, Kirvar E, Wilkie G, et al. Bos taurus and Bos indicus (Sahiwal) calves respond differently to infection with Theileria annulata and produce markedly different levels of acute phase proteins. Int J Parasitol. 2005;35:337-47.

23. Hunter RA, Siebert BD. Utilization of low-quality roughage by Bos-taurus and Bos-indicus cattle .1. rumen digestion. Brit J Nutr. 1985;53:637-48.

24. Gaughan JB, Mader TL, Holt SM, Sullivan ML, Hahn GL. Assessing the heat tolerance of 17 beef cattle genotypes. Int $\mathbf{J}$ Biometeorol. 2010;54:617-27.

25. Bishop SC, Morris CA. Genetics of disease resistance in sheep and goats. Small Rumin Res. 2007;70:48-59.

26. Zwald NR, Weigel KA, Chang YM, Welper RD, Clay JS. Genetic selection for health traits using producer-recorded data. I. Incidence rates, heritability estimates, and sire breeding values. J Dairy Sci. 2004;87:4287-94.

27. Rezk MA, Ponzoni RW, Khaw HL, Kamel E, Dawood T, John G. Selective breeding for increased body weight in a synthetic breed of Egyptian Nile tilapia, Oreochromis niloticus: response to selection and genetic parameters. Aquaculture. 2009;293:187-94.

28. Gensollen T, Iyer SS, Kasper DL, Blumberg RS. How colonization by microbiota in early life shapes the immune system. Science. 2016;352:539-44.

29. Greenwood PL, Cafe LM. Prenatal and pre-weaning growth and nutrition of cattle: long-term consequences for beef production. Animal. 2007;1:1283-96.

30. Tamburini S, Shen N, Wu HC, Clemente JC. The microbiome in early life: implications for health outcomes. Nat Med. 2016;22:713-22.

31. Elzo MA, West RL, Johnson DD, Wakeman DL. Genetic variation and prediction of additive and nonadditive genetic effects for six carcass traits in an Angus-Brahman multibreed herd. J Anim Sci. 1998;76:1810-23.

32. Mir RA, Weppelmann TA, Teng L, Kirpich A, Elzo MA, Driver $\mathrm{JD}$, et al. Colonization dynamics of cefotaxime resistant bacteria in beef cattle raised without cephalosporin antibiotics. Front Microbiol. 2018;9:500.

33. Kozich JJ, Westcott SL, Baxter NT, Highlander SK, Schloss PD. Development of a dual-index sequencing strategy and curation pipeline for analyzing amplicon sequence data on the MiSeq
Illumina sequencing platform. Appl Environ Micro. 2013;79:5112-20.

34. Geng J, Song Q, Tang X, Liang X, Fan H, Peng H, et al. Cooccurrence of driver and passenger bacteria in human colorectal cancer. Gut Pathog. 2014;6:26.

35. Langille MGI, Zaneveld J, Caporaso JG, McDonald D, Knights D, Reyes JA, et al. Predictive functional profiling of microbial communities using 16S rRNA marker gene sequences. Nat Biotechnol. 2013;3:814.

36. Gobena M, Elzo MA, Mateescu RG. Population structure and genomic breed composition in an angus-brahman crossbred cattle population. Front Genet. 2018;9:90.

37. Lopez R, Thomas MG, Hallford DM, Keisler DH, Silver GA, Obeidat BS, et al. Metabolic hormone profiles and evaluation of associations of metabolic hormones with body fat and reproductive characteristics of Angus, Brangus, and Brahman heifers. Prof Anim Sci 2006;22:273-82.

38. Chase CC Jr., Chenoweth PJ, Larsen RE, Hammond AC, Olson TA, West RL, et al. Growth, puberty, and carcass characteristics of Brahman-, Senepol-, and Tuli-sired F1 Angus bulls. J Anim Sci. 2001;79:2006-15.

39. Piper EK, Jonsson NN, Gondro C, Lew-Tabor AE, Moolhuijzen $\mathrm{P}$, Vance ME, et al. Immunological profiles of Bos taurus and Bos indicus cattle infested with the cattle tick, Rhipicephalus (Boophilus) microplus. Clin Vaccin Immunol. 2009; 16:1074-86.

40. Nylund L, Nermes M, Isolauri E, Salminen S, de Vos WM, Satokari R. Severity of atopic disease inversely correlates with intestinal microbiota diversity and butyrate-producing bacteria. Allergy. 2015;70:241-4.

41. Machiels K, Joossens M, Sabino J, De Preter V, Arijs I, Eeckhaut $\mathrm{V}$, et al. A decrease of the butyrate-producing species Roseburia hominis and Faecalibacterium prausnitzii defines dysbiosis in patients with ulcerative colitis. Gut. 2014;63:1275-83.

42. Takahashi K, Nishida A, Fujimoto T, Fujii M, Shioya M, Imaeda $\mathrm{H}$, et al. Reduced abundance of butyrate-producing bacteria species in the fecal microbial community in Crohn's disease. Digestion. 2016;93:59-65.

43. Ohkawara S, Furuya H, Nagashima K, Asanuma N, Hino T. Oral administration of Butyrivibrio fibrisolvens, a butyrate-producing bacterium, decreases the formation of aberrant crypt foci in the colon and rectum of mice. J Nutr. 2005;135:2878-83.

44. Uhde FL, Kaufmann T, Sager H, Albini S, Zanoni R, Schelling E, et al. Prevalence of four enteropathogens in the faeces of young diarrhoeic dairy calves in Switzerland. Vet Rec. 2008;163:362-6.

45. Gomez DE, Arroyo LG, Costa MC, Viel L, Weese JS. Characterization of the fecal bacterial microbiota of healthy and diarrheic dairy calves. J Vet Intern Med. 2017;31:928-39.

46. Turner JR. Intestinal mucosal barrier function in health and disease. Nat Rev Immunol. 2009;9:799-809.

47. Desai MS, Seekatz AM, Koropatkin NM, Kamada N, Hickey CA, Wolter $\mathrm{M}$, et al. A dietary fiber-deprived gut microbiota degrades the colonic mucus barrier and enhances pathogen susceptibility. Cell. 2016;167:1339-53.

48. Hoorens PR, Rinaldi M, Li RW, Goddeeris B, Claerebout E, Vercruysse $\mathrm{J}$, et al. Genome wide analysis of the bovine mucin genes and their gastrointestinal transcription profile. Bmc Genomics. 2011;12:140.

49. Van de Merwe JP, Stegeman JH, Hazenberg MP. The resident faecal flora is determined by genetic characteristics of the host. Implications for Crohn's disease? Antonie Van Leeuwenhoek. 1983;49:119-24.

50. Khachatryan ZA, Ktsoyan ZA, Manukyan GP, Kelly D, Ghazaryan KA, Aminov RI. Predominant role of host genetics in controlling the composition of gut microbiota. PLoS One. 2008;3: e3064. 
51. Blekhman R, Goodrich JK, Huang K, Sun Q, Bukowski R, Bell JT, et al. Host genetic variation impacts microbiome composition across human body sites. Genome Biol. 2015;16:191.

52. Mir RA, Weppelmann TA, Johnson JA, Archer D, Morris JG Jr., Jeong KC. Identification and characterization of cefotaxime resistant bacteria in beef cattle. PLoS One. 2016;11:e0163279.

53. Hernandez-Sanabria E, Goonewardene LA, Wang Z, Zhou M, Moore SS. Influence of sire breed on the interplay among rumen microbial populations inhabiting the rumen liquid of the progeny in beef cattle. PLoS One. 2013;8:e58461.

54. Smith KE, Stenzel SA, Bender JB, Wagstrom E, Soderlund D, Leano FT, et al. Outbreaks of enteric infections caused by multiple pathogens associated with calves at a farm day camp. Pediatr Infect Dis J. 2004;23:1098-104.

55. Talukder KA, Aslam M, Islam Z, Azmi IJ, Dutta DK, Hossain S, et al. Prevalence of virulence genes and cytolethal distending toxin production in Campylobacter jejuni isolates from diarrheal patients in Bangladesh. J Clin Microbiol. 2008;46:1485-8.

56. Ferrarezi MC, Cardoso TC, Dutra IS. Genotyping of Clostridium perfringens isolated from calves with neonatal diarrhea. Anaerobe 2008;14:328-31.

57. McGuckin MA, Linden SK, Sutton P, Florin TH. Mucin dynamics and enteric pathogens. Nat Rev Microbiol. 2011;9:265-78.

58. Linden SK, Sutton P, Karlsson NG, Korolik V, McGuckin MA. Mucins in the mucosal barrier to infection. Mucosal Immunol. 2008;1:183-97.

59. Pelaseyed T, Bergstrom JH, Gustafsson JK, Ermund A, Birchenough GM, Schutte A, et al. The mucus and mucins of the goblet cells and enterocytes provide the first defense line of the gastrointestinal tract and interact with the immune system. Immunol Rev. 2014;260:8-20.

60. Tailford LE, Crost EH, Kavanaugh D, Juge N. Mucin glycan foraging in the human gut microbiome. Front Genet. 2015;6:81.

61. Hollingsworth MA, Swanson BJ. Mucins in cancer: protection and control of the cell surface. Nat Rev Cancer. 2004;4:45-60.

62. Yamamoto-Furusho JK, Ascano-Gutierrrez I, FuruzawaCarballeda J, Fonseca-Camarillo G Differential expression ofmuc12, muc16, and muc20 in patients with active and remission ulcerative colitis. Mediat Inflamm. 2015;2015:659018.

63. Sheng YH, Hasnain SZ, Florin TH, McGuckin MA. Mucins in inflammatory bowel diseases and colorectal cancer. J Gastroenterol Hepatol. 2012;27:28-38.

64. Kato D, Suzuki Y, Haga S, So K, Yamauchi E, Nakano M, et al. Utilization of digital differential display to identify differentially expressed genes related to rumen development. Anim Sci J. 2016;87:584-90.
65. Steele MA, Penner GB, Chaucheyras-Durand F, Guan LL. Development and physiology of the rumen and the lower gut: Targets for improving gut health. J Dairy Sci. 2016; 99:4955-66.

66. Lozupone CA, Stombaugh JI, Gordon JI, Jansson JK, Knight R. Diversity, stability and resilience of the human gut microbiota. Nature. 2012;489:220-30.

67. Sokol H, Pigneur B, Watterlot L, Lakhdari O, Bermudez-Humaran LG, Gratadoux JJ, et al. Faecalibacterium prausnitzii is an antiinflammatory commensal bacterium identified by gut microbiota analysis of Crohn disease patients. P Natl Acad Sci Usa 2008;105:16731-13676.

68. Foditsch C, Pereira RV, Ganda EK, Gomez MS, Marques EC, Santin T, et al. Oral administration of Faecalibacterium prausnitzii decreased the incidence of severe diarrhea and related mortality rate and increased weight gain in preweaned dairy heifers. PLoS One. 2015;10:e0145485.

69. Jenq RR, Taur Y, Devlin SM, Ponce DM, Goldberg JD, Ahr KF, et al. Intestinal Blautia is associated with reduced death from graft-versus-host disease. Biol Blood Marrow Tr. 2015;21:1373-83.

70. Berni Canani R, Sangwan N, Stefka AT, Nocerino R, Paparo L, Aitoro R, et al. Lactobacillus rhamnosus GG-supplemented formula expands butyrate-producing bacterial strains in food allergic infants. Isme J. 2016;10:742-50.

71. Elzo MA, Johnson DD, Wasdin JG, Driver JD. Carcass and meat palatability breed differences and heterosis effects in an AngusBrahman multibreed population. Meat Sci. 2012;90:87-92.

72. Robbins JB, Schneerson R, Szu SC. Perspective - hypothesis serum igg antibody is sufficient to confer protection against infectious-diseases by inactivating the inoculum. J Infect Dis. 1995;171:1387-98.

73. Shyma KP, Gupta JP, Singh V. Breeding strategies for tick resistance in tropical cattle: a sustainable approach for tick control. J Parasit Dis 2015;39:1-6.

74. Mir RA, Weppelmann TA, Kang M, Bliss TM, DiLorenzo N, Lamb GC, et al. Association between animal age and the prevalence of Shiga toxin-producing Escherichia coli in a cohort of beef cattle. Vet Microbiol. 2015;175:325-31.

75. Ba Q, Li M, Chen P, Huang C, Duan X, Lu L, et al. Sexdependent effects of cadmium exposure in early life on gut microbiota and fat accumulation in mice. Environ Health Perspect. 2017;125:437-46.

76. Org E, Mehrabian M, Parks BW, Shipkova P, Liu X, Drake TA, et al. Sex differences and hormonal effects on gut microbiota composition in mice. Gut Microbes. 2016;7:313-22. 